\title{
A Variable Condition Calculation Method for Thermal Power Units Sliding Pressure Operation
}

\author{
Wei Liu, Jizhen Liu, Wei Wang, Deliang Zeng, and Taihua Chang
}

\begin{abstract}
This paper first analyzes how thermodynamic system state parameters are affected when main steam's pressure changes under sliding pressure operation .Then a new state distribution equation of sliding pressure operation based on thermo-economy equation of state of thermal power unit is given.With the help of this equation, a new algorithm is proposed to determine the state parameters distribution for variable working condition under sliding pressure operation. Experiment of one supercritical unit is provided to validate the effectiveness of our approach under sliding pressure operation. Simulation results show that this algorithm is beneficial for analyzing the security and economy of thermal power unit with deep fast variable loads. It also has vital important significance for thermal power unit to develop its peak shaving ability.
\end{abstract}

Index Terms - Sliding pressure, variable working ondition, thermo-economy, thermal power unit.

\section{INTRODUCTION}

With the development in the structure of residential electricity consumption, peak-valley difference of Electric Power Systems is increasing. To improve the ability of in depth peak load cycling is necessary, which requires thermal power units could be under sliding pressure operation in long-term state and the capacity of adjustment under fast load variation. In order to analyze the economic and security of thermal power units under load variation, a status parameter distribution in overall working conditions is essential. However, there is still no accurate method to obtain the parameters. Moreover, sometimes the units couldn't be under design conditions because of the complexity of the plant operation and the aging equipment. Therefore, A Variable Condition Calculation method for Thermal power units under sliding pressure operation is proposed, which is not only beneficial for analyzing the security and economy of thermal power unit with deep fast variable loads, but also has vital important significance for thermal power unit to develop its peak shaving ability.

In this paper, on the basis of the Thermal Economic State Equation of thermal power plants, variable conditions theory and analysis of Influence between the various state parameters, propose a variable condition calculation method for thermal power units under sliding pressure operation, through the algorithm a Real-time calculation of

Manuscript received October 18, 2012; revised November 23, 2012.

Wei Liu, Wei Wang, Deliang Zeng, and Taihuang Chang are with North China Electric Power University,Beijing,102206, China (e-mail: 1w2359@163.com)

Jizhen Liu was with Huazhong University of Science \& Technology. Wuhan, 430074 China. He is now with North China Electric Power University, Beijing, 102206, China. the economy and security of units is obtained.

\section{THERMO-ECONOMY EQUATION OF STATE OF THERMAL POWER UNIT}

Based on the main loop structure of thermodynamic system, Thermo-economy equation of state is a group of multiple linear equations which are summarized by conservations of energy and mass. Indices of equation are determined by the equipment structure and state of thermodynamic system, as in [1].

\section{A. Steam-Water Distribution Equation}

Reference [2] shows Steam-water distribution general equation is given by:

$$
[A]\left[D_{i}\right]+\left[Q_{f i}\right]=D_{0}\left[\tau_{i}\right]
$$

where $\left[D_{i}\right]$ is a vector and consist with steam's flow of each stage; $\left[\tau_{i}\right]$ is a vector and consist with feed-water enthalpy rise of heater in different level; $\tau_{i}$ denotes the feed-water enthalpy of the ith stage heater and is defined as $\tau_{i}=h_{w i}-h_{w(i+1)} ;[A]$ is system matrix.

\section{B. System Heat Absorption Equation}

System heat absorption equation is given by

$$
\begin{aligned}
& Q=D_{0}\left(h_{0}+\sigma-h_{f w}\right)-\sum_{i=1}^{2} D_{i} \sigma-\left[D_{u}\right]_{c}^{T}[\sigma]_{c} \\
& +D_{b l}\left(h_{b l}-h_{f w}\right)+D_{s s}\left(h_{f w}-h_{s s}\right)+D_{r s}\left(h_{r h}-h_{r s}\right)
\end{aligned}
$$

where $\sigma$ is the unit reheat endothermic variable, $D_{b l}, h_{b l}$ are system sewage disposal flow and enthalpy, $D_{s s}, h_{s s}$ are temperature reduction water flow and enthalpy for the superheaters, $h_{f w}$ is feed-water enthalpy.

\section{System Output Working Equation}

The output power equation of whole system cycling is given by:

$$
\begin{aligned}
& N=D_{0}\left(h_{0}+\sigma-h_{c}\right)- \\
& \sum_{i=1}^{2} D_{i}\left(h_{i}+\sigma-h_{c}\right)-\sum_{i=3}^{8} D_{i}\left(h_{i}-h_{c}\right) \\
& -\left[D_{u}^{\prime}\right]^{T}\left[h_{u}^{\prime}\right]+D_{r s}\left(h_{r h}-h_{c}\right)
\end{aligned}
$$

where $h_{c}$ is exhaust enthalpy, $\left[D_{u}^{\prime}\right]^{T},\left[h_{u}^{\prime}\right]$ are auxiliary steam-water flow and enthalpy which depart from turbine. 


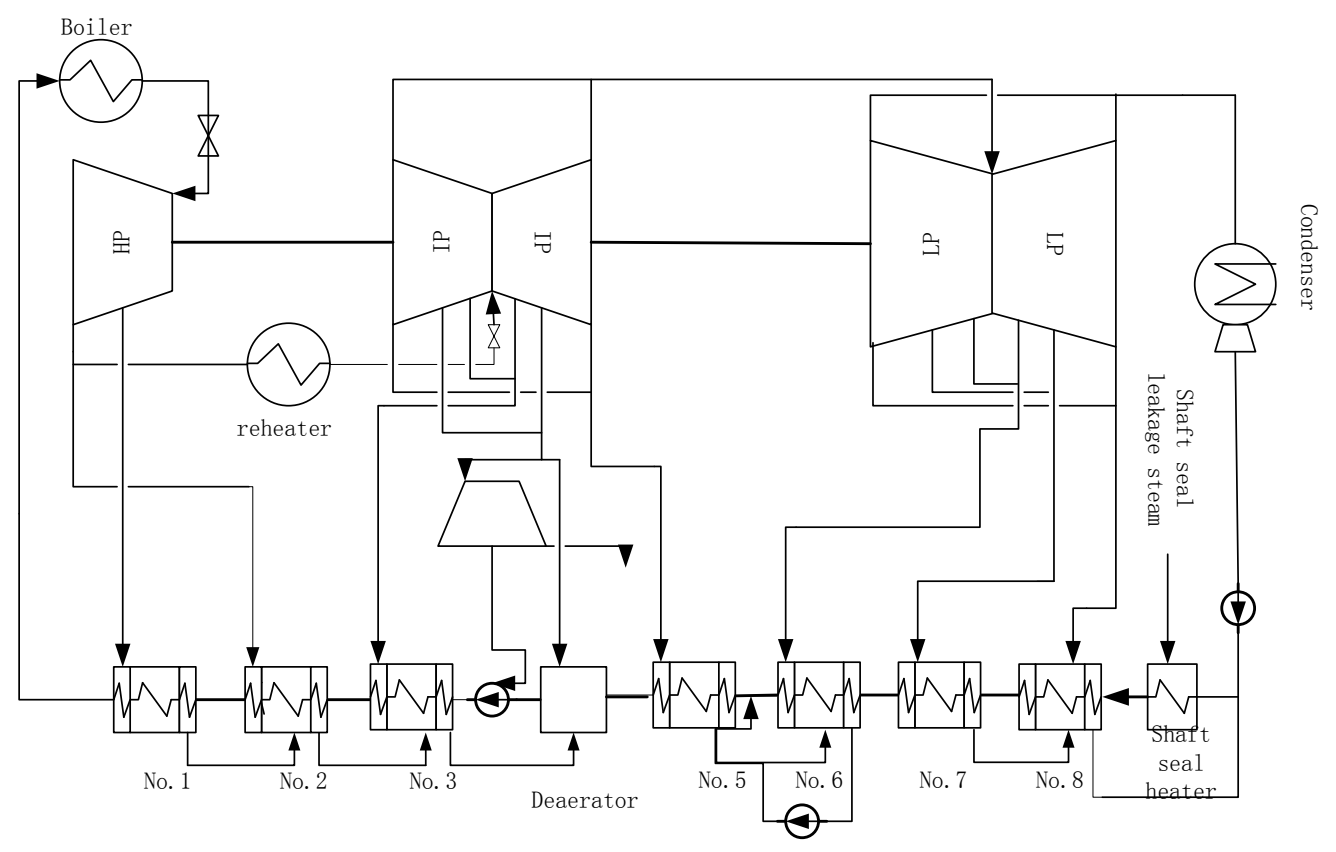

Fig. 1. Structure of a general ultra supercritical unit

\section{VARIABLE WORKING CONDITION ANALYSIS OF THERMAL POWER UNITS UNDER SLIDING PRESSURE OPERATION}

On the assumption that main steam's temperature, reheat steam's temperature and the exhaust pressure of units are constant. If the main steam's pressure change, the steam admission's state of turbine will change, which result in variation of the overall state parameter distributions. Elements of matrix [A] have one-to-one relationships with state parameters of the thermal system in [3]. Therefore, it's difficult to calculate the unit's thermo-economy with the thermal economic state equation. In order to determine the status parameters' distribution and figure out the change of system matrix [A], it is needed to analyze the whole heat cycling structures under variable working condition. The structure of a general ultra supercritical unit, N100026.25/600/600 is shown in Fig. 1.

\section{A. The Effect of Alerting Main Steam's Pressure on Main Steam's Flow Quantities}

The valve opening in governing stage is constant, so that the governing stage's flow area is invariant, which is the applicable condition of Frugal formula, so that the relation between main steam's pressure and main steam's flow is expressed by

$$
\frac{D_{0 l}}{D_{0}}=\frac{\sqrt{p_{0 l}^{2}-p_{c l}^{2}}}{\sqrt{p_{0}^{2}-p_{c 0}^{2}}}
$$

where $D_{0}, D_{0 l}$ are main steam's flow of standard working condition and variable working condition; $p_{0}, p_{0 l}$ are main steam's pressure of standard working condition and variable working condition; $p_{c l}, p_{c 0}$ are back pressure of standard working condition and variable working condition.
B. The Effect of Alerting Main Steam's Pressure on Flow Quantities of Steam Extracted at the Various Stages

The governing stage' flow area is constant under slide pressure mode, governing stage and The Extraction Steam stages No. 1 can be seen as one controlled body, the other steam extraction grades Separately as controlled bodies. Assume ${ }^{\left[\alpha_{i}\right]}$ a vector consist with the shares of steam extracted at the various stages, it can be expressed by

$$
\left[D_{r l i}\right]=D_{0 l}\left[\alpha_{i}\right]
$$

where $\left[D_{r l i}\right]$ denotes a vector consist with the flow quantities of steam extracted at the various stages, the steam extraction's pressure can be expressed by

$$
p_{r l i}=p_{r 0 i} \frac{D_{r l i}}{D_{r 0 i}}
$$

where $D_{r 0 i}$ denotes flow quantities of steam extracted at the stage $\mathrm{i}$ under the standard condition; $p_{r 0 i}$ is the pressure of steam extracted at the stage $i$ under the standard condition.

\section{The Effect of Alerting Main Steam's Pressure on Enthalpy of Steam Extracted at the Various Stages and Exhaust Enthalpy of Unit.}

Based on the Theory of Variable Conditions, Relative internal efficiency of intermediate stages in non-wet steam region, can be seen as Constants, which is expressed by

$$
\eta_{i-1, i}=\frac{h_{i-1}-h_{i}}{h_{i-1}-h_{i s}}
$$

where $\eta_{i-1, i}$ denotes the relative internal efficiency of stage $\mathrm{i} ; h_{i s}$ denotes the ith stage's ideal extraction steam 
enthalpy; $h_{i}$ is extraction steam enthalpy of stage $i$, which is calculated as

$$
h_{i}=h_{i-1}-\eta_{i-1, i}\left(h_{i-1}-h_{i s}\right)
$$

In this premise that main steam's temperature is constant no matter how main steam's pressure change, the inlet of turbine can be seen as the extremity of stage 0.Through above analysis, the effect of alerting main steam's pressure on enthalpy of steam in the overheated state can be achieved.

Generally, last few stages of Low-pressure cylinder is in wet steam region, so that these Relative internal efficiency isn't as constant as the others do. Making use of Pressure ratio pi/pi-1, a fitting Straight Line for Relative internal efficiency of LP-cylinder can be obtained, as in [4], which is helpful to calculate the enthalpy of extraction wet steam with [5](exhaust steam can be seen as 9th extraction steam).

\section{The Effect of Alerting Main Steam's Pressure on Enthalpy of Water Enthalpy Trough Heaters}

The terminal temperature differences of heater and the pressure loss of each steam extraction pipe may remain stable when unit is under normal operation. Each steam extraction pressure may change, furthermore, their corresponding saturated water pressure may also change when main steam's pressure changes. Meanwhile, outlet water enthalpy and drain water enthalpy are affected by the terminal temperature differences. Reference [6] shows quantitative relationship between extraction steam's pressure and terminal temperature difference

Fig. 1 shows that $i+1$ th stage heater outlet enthalpy equals $i$ th stage inlet enthalpy (due to pump power, enthalpy between No.3 and No.4 is not as same as enthalpy between No.5 and No.6). For the loss of pipe pressure is constant, it exist following formula

$$
p_{r l i}^{\prime}=\left(1-\Delta p_{i}\right) p_{r l i}
$$

where $p_{r l i}^{\prime}$ is steam admission's pressure of the ith stage heater, $M P a ; \Delta p_{i}$ is the loss of pipe pressure between ith stage steam extraction and heater steam inlet. The outlet temperature of heater $t_{w(i+1)}$ is given by

$$
t_{w(i+1)}=t_{s i}-\Delta t_{s i}
$$

where $t_{s i}$ is saturated water temperature under ith heater admission pressure, ${ }^{\circ} \mathrm{C}$, it can be calculated by $p_{r l i} ; \Delta t_{s i}$ is the upper terminal temperature difference of ith heater.

Heater dewatering temperature $t_{d i}$ is given by:

$$
t_{d i}=t_{w i}+\Delta t_{x i}
$$

where $\Delta t_{x i}$ is the ith heater upper terminal temperature difference, ${ }^{\circ} \mathrm{C}$. Pressure has limited effect on unsaturated water's enthalpy, therefore, heater outlet enthalpy and dewatering enthalpy can be derived from temperature.

\section{ITERATIVE STRATEGY}

Regard condenser as start point, analyzing the changes of each parameter stage-by-stage. When main steam's pressure changes, backpressure stays the same, so does condenser outlet water temperature. The heat provided by condensate pump and gland heater is closed related to main steam's flow. By fitting main steam's flow to $\Delta D_{f t}^{0}$, it can be sure that how pure heat changes when main steam's pressure changes. Due to energy conservation, for the enthalpy of No8 heater, it exist

$$
\left(1-\sum_{i=1}^{6} \alpha_{i}\right) h_{c}^{\prime}+\Delta D_{f}^{0}=\left(1-\sum_{i=1}^{6} \alpha_{i}\right) h_{w 8}
$$

where $h_{c}^{\prime}$ denotes condensation water enthalpy, $k J / k g$

Through above variable working condition analysis, the realization of variable condition calculation need a standard condition, which provide exact parameters for a start point to iterative calculation. Fig. 2 shows what the iterative strategy is consider the heat absorption capacity and working capacity, relative inner efficiency of turbine is given by

$$
\eta=\frac{N}{Q} \times 100 \%
$$

where $N$ denotes output power of whole system cycling; $Q$ denotes system heat absorption.

The steam consumption is given by

$$
q_{q}=\frac{3600 D_{0 l}}{N \eta_{g}}
$$

The heat consumption is given by

$$
q_{r}=\frac{3600}{\eta \eta_{g}}
$$

where $\eta_{g}$ is efficiency of generation?

\begin{tabular}{|c|c|c|c|c|c|c|c|c|}
\hline & $\alpha_{1}$ & $\alpha_{2}$ & $\alpha_{3}$ & $\alpha_{4}$ & $\alpha_{5}$ & $\alpha_{6}$ & $\alpha_{7}$ & $\alpha_{8}$ \\
\hline $1 \mathrm{st}$ & 0.03791 & 0.09173 & 0.0357 & 0.07681 & 0.03945 & 0.04445 & 0.02682 & 0.0207 \\
\hline 2nd & 0.03676 & 0.09193 & 0.03692 & 0.07266 & 0.03948 & 0.04458 & 0.02664 & 0.02162 \\
\hline 3nd & 0.03679 & 0.09206 & 0.0369 & 0.07142 & 0.0395 & 0.04459 & 0.02669 & 0.02174 \\
\hline 4 th & 0.0368 & 0.09206 & 0.03689 & 0.07107 & 0.03951 & 0.04459 & 0.02669 & 0.02178 \\
\hline 5 th & 0.0368 & 0.09205 & 0.03689 & 0.07096 & 0.03951 & 0.0446 & 0.0267 & 0.02179 \\
\hline 6th & 0.0368 & 0.09205 & 0.03689 & 0.07092 & 0.03951 & 0.0446 & 0.0267 & 0.02179 \\
\hline
\end{tabular}

\section{EXAMPLES}

Applying a certain Ultra-supercritical unit, no N1000$26.25 / 600 / 600$, taking the $75 \%$ load condition as standard condition, which is the initial iteration state, how $\left[\alpha_{i}\right]$ 's iteration processing when the main steam's pressure is changing $(15.7 \mathrm{mpa})$ is show in Table I.

TABLE I: RESULTS OF ITERATIVE CALCULATION 


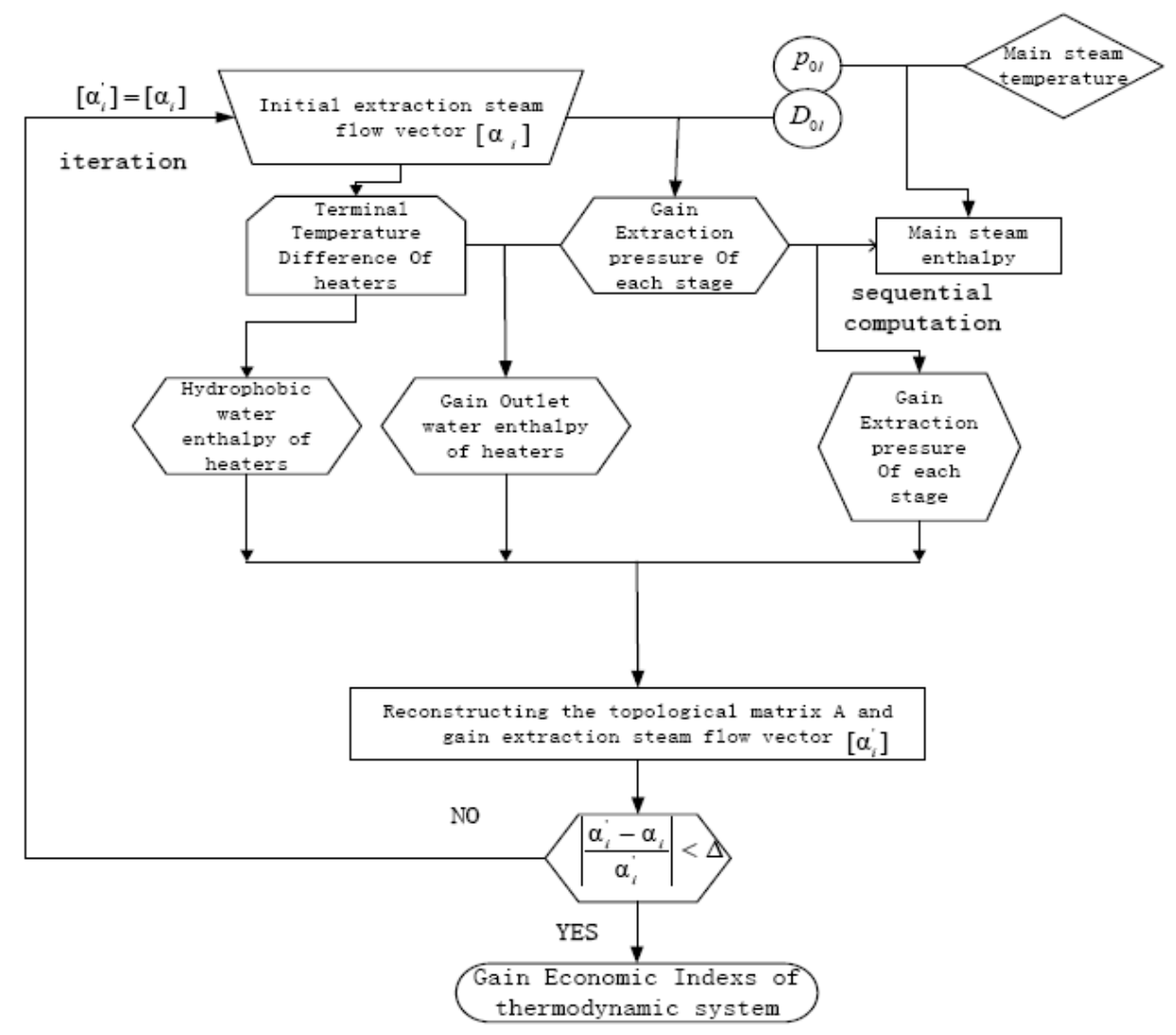

Fig. 2. Iterative strategy

Table I shows that the maximum relative error is $0.08 \%$ after the sixth iteration, it's distinct that the new method have an excellent convergence, the heat economy index can be calculated as Fig. 2 and Fig. 3

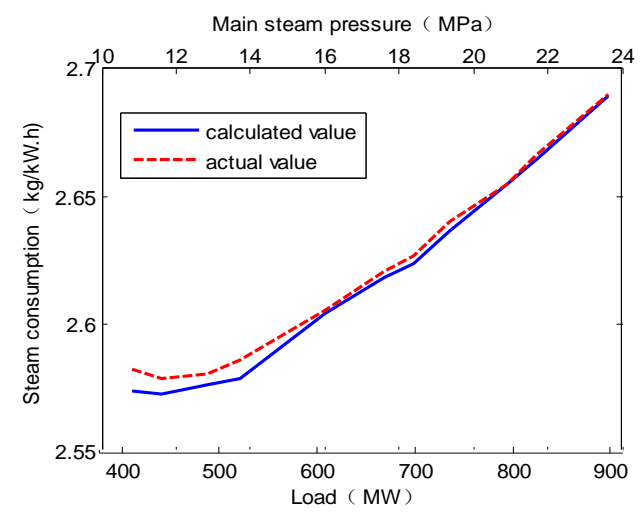

Fig. 3. CORRELATION CURVE for steam consumption of thermodynamic system

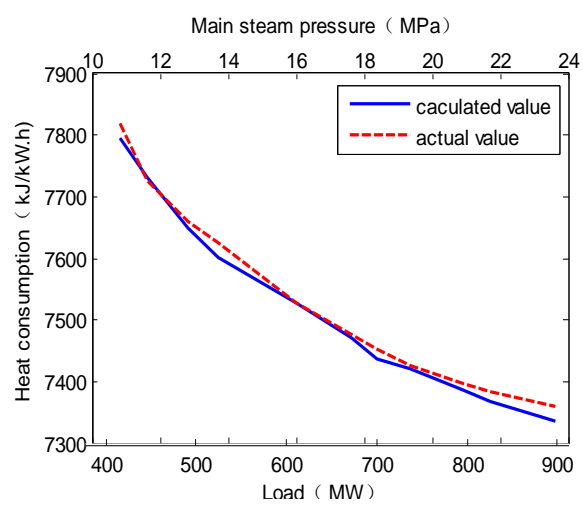

Fig. 4. CORRELATION CURVE for heat consumption of thermodynamic system
Fig. 3 and Fig. 4 show that in the interval of $40 \%$-load to $90 \%$-load, the maximum relative errors of steam consumption and heat consumption $0.3267 \%$ and $0.3317 \%$, respectively, which prove the effectiveness of the method.

\section{SUMMARIES}

This paper makes a detailed analysis of the thermal power units under variable condition, to calculate the heat economy index through reconstructing the topological matrix A of thermodynamic system and iterative calculation. A example applying a 1000MW unit shows that new method has the advantages including excellent convergence, high precision, easy to implement and so on. Furthermore, this method provide a useful prediction of the thermal economy's index for Staffs when they try changing some parameters of thermodynamic system,including overall distribution of thermodynamic system'state parameters, which has big influence on developing thermal power plant's peak shaving ability and security.

\section{ACKNOWLEDGMENT}

This paper is projected by National Key Basic Research Program of China (2012CB215203) and National Natural Science Foundation of China (51036002).

\section{REFERENCES}

[1] J. L. Guo, S. F. Zhang, Z. P. Song, and H. P. Chen, "A matrix method for thermal system analysis in a power plant," IEEE Trans. Proceedings of the CSEE, vol. 24, no. 1, pp. 205-210, 2004.

[2] S. L. Yan, C. F. Zhang, Y. H. Li, and X. C. Chao, "The Steam-water Distribution General Matrix Equation of Thermal System for The Coal-fired Power Unit," IEEE Trans. Proceedings of the CSEE, vol. 20, pp. 69-73, 2000. 
[3] C. F. Zhang, L. P. Li, and M. Z. Zhang, "Steam-Water distribution standard equation of thermo-system for coal-fired power plant," in Proc. of International conference on Power Engineering, Japan, 2003.

[4] W. Wang, D. L. Zeng, T. T. Yang, Z. G. Zhang, and J. Z. Liu, "The Optimum Running of Circulating Water Pumps Based on Estimated Condenser Pressure," IEEE Trans. Proceedings of the CSEE, vol. 30, no. 14 , pp. 7-12,2010.

[5] J. Qian, P. H. Wang, H. Zhao, and X. Y. Peng, "Research of Realtime Monitoring Model forCalculating Exhaust Enthalpy," in Proc. Power and Energy Engineering Conference (APPEEC), Asia-Pacific, 2010.

[6] S. L. Yan, Y. H. Li, and Q. Y. Wu, "Strength Coefficient Calculation Model of Influence of Main Steam Temperature Variation on Coal Consumption Rate," IEEE Trans. Proceedings of the CSEE, vol. 31, no. 26 , pp. 38-43, 2011.

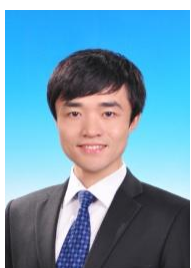

Wei Liu was born in Chenzhou City, Hunan Province, China. Aug, 7, 1988. Wei Liu is a postgraduate (since 2010), Master's degree in control theory and control engineering, in North China electric power university, Beijing, China. Major in modeling for thermodynamic systems and state analysis of thermal power plants.

$\mathrm{He}$ is studying at North China electric power university for Master, who has gotten a special scholarship and the title of outstanding graduate cadres there. His Current research interest is state analysis of thermal power plants

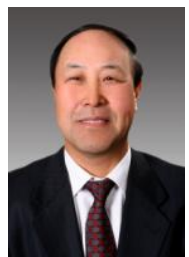

Jizhen Liu was born in Lvliang City, Shanxi Province, China. Aug, 7, 1951. Jizhen Liu earned he doctor's degree from Huazhong University of Science and Technology, Wuhan, China, in 2007. And his research is mainly in respect of Modeling and Intelligent Control on Large capacity units and Supervisory information system in plant level. $\mathrm{He}$ is now working in North China Electric Power University as the headmaster, Ph.D. supervisor and Professor. He and his research team has finished more than 20 Key research projects for National and enterprise, published more than 160 scientific papers. Now he is chief scientist in the State Key Laboratory: new energy power system. Prof. Liu is IEE fellow, member of the Electricity Regulatory Commission, vice chairman in China Institute of Power Engineering, Executive director of Chinese society for Electrical Engineering, etc.

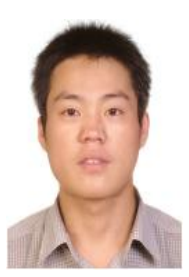

Wei Wang was born in Linyi City, Shandong Province, China. 1986. Wei Wang received the doctor's degree from North China Electric Power University, Beijing, China, in 2011. And his research is mainly in respect of energy saving and optimization on power systems. He is now working in North China Electric Power University as an instructor. And he is a scientist in National Key Basic Research Program of China (2012CB215203) and National Natural Science Foundation of China (51036002). His current interest is New energy power system.

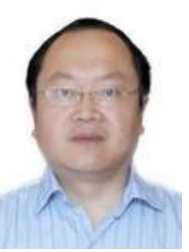

Deliang Zeng was born in Nov, 9, 1969. He earned the doctor's degree from North China Electric Power University, Beijing, China, in 2000. And his research is mainly in respect of Modeling and Intelligent Control on Thermal engineering. He is now working in North China Electric Power University as a Associate Professor. He earned the China Power Science and Technology Award second prize in 2003, first prize in 2005. And he is a scientist in National Key Basic Research Program of China (2012CB215203) and National Natural Science Foundation of China (51036002). His current interest is new energy power system.

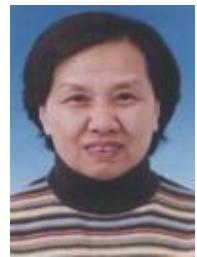

Taihuang Chang was born in Taiyuan City, Shanxi Province, China. Feb. 1951. Taihuang Chang received Bachelor degree from North China Electric Power University, Baoding, China, in 1976. And her research is mainly in respect of Data Mining and Information Fusion.vShe is now working in North China Electric Power University as a Professor. She earned The Beijing Universities excellent moral educators and Annual advanced personal in 2005, educators morality advanced individual in 2006, etc. Now her current interest is Complex thermodynamic system analysis. 